\title{
Targeted TLR9-activation/STAT3-blocking abrogates immunosuppressive functions of myeloid-derived suppressor cells from late-stage prostate cancer patients
}

\author{
Dewan Md Sakib Hossain*, Dayson Moreira, Qifang Zhang, Stephen J Forman, Sumanta Pal, Marcin Kortylewski
}

From Society for Immunotherapy of Cancer 29th Annual Meeting

National Harbor, MD, USA. 6-9 November 2014

Recent clinical advances underscored that elimination of tumor-induced immunosuppression is prerequisite for successful therapy of advanced human cancers. The myeloid-derived suppressor cells (MDSCs) are one of the major populations limiting $\mathrm{T}$ cell-mediated antitumor immune responses in late-stage solid tumors, such as castration-resistant prostate cancers (CRPCs). Targeting MDSCs proved challenging due to their heterogeneity and phenotypic similarities with other myeloid cell lineages. Here, we identify a population of granulocytic MDSCs, which accumulate in patients' blood during progression of prostate cancer from localized to metastatic disease. The CRPC-associated MDSCs potently inhibit proliferation, IFN- $\gamma$ and Granzyme-B production in autologous $\mathrm{CD}^{+} \mathrm{T}$ cells. Importantly, the MDSCs in blood and in lymph nodes of CRPC patients show high levels of activated STAT3 transcription factor, which is a crucial immune checkpoint regulator. We previously generated an original method to silence genes specifically in $\mathrm{TLR}^{+}$myeloid cells using CpG-siRNA conjugates $[1,2]$. Now, we demonstrate that the CpG-STAT3siRNA is also effectively internalized by human MDSCs and induces STAT3 silencing without the need for transfection reagents. Our further studies demonstrated that twopronged TLR9-activation/STAT3-blocking by CpGSTAT3siRNA inhibits immunosuppressive effects of CRPC-MDSCs on $\mathrm{CD}^{+} \mathrm{T}$ cells, while both TLR9 triggering or STAT3 inhibition alone have only minimal effects. The reversal of MDSC immunosuppression by CpGSTAT3 siRNA results from reduced expression and enzymatic activity of Arginase I, a downstream STAT3 target gene. Overall, our study demonstrates the feasibility of overcoming immunosuppression in CRPC patients using CpG-STAT3siRNA strategy to alleviate effects of MDSCs. We anticipate that our strategy to reverse MDSCmediated immunosuppression without depleting myeloid cell populations will generate safer and more effective immunotherapy for late-stage, metastatic prostate cancers and potentially other solid tumors.

This project described was supported by the National Cancer Institute of the National Institutes of Health under award number R01CA155367 and by the Department of Defense Prostate Cancer Research Program under award number W81XWH-12-1-0132 to M.K.

Published: 6 November 2014

\section{References}

1. Kortylewski $M$, Swiderski $P$, Herrmann $A$, et al: In vivo delivery of siRNA to immune cells by conjugation to a TLR9 agonist enhances antitumor immune responses. Nat Biotechnol 2009, 27:925-932.

2. Zhang Q, Hossain DM, Nechaev S, et al: TLR9-mediated siRNA delivery for targeting of normal and malignant human hematopoietic cells in vivo. Blood 2013, 121:1304-1315.

\section{doi:10.1186/2051-1426-2-S3-P104}

Cite this article as: Md Sakib Hossain et al:: Targeted TLR9-activation/ STAT3-blocking abrogates immunosuppressive functions of myeloidderived suppressor cells from late-stage prostate cancer patients. Journal for ImmunoTherapy of Cancer 2014 2(Suppl 3):P104. 